\title{
Research Article \\ On Modular Ball-Quotient Surfaces of Kodaira Dimension One
}

\begin{abstract}
Aleksander Momot
Departement Mathematik, ETH Zürich, HG J65, Rämistraße 101, 8092 Zürich, Switzerland

Correspondence should be addressed to Aleksander Momot, aleksander.momot@math.ethz.ch

Received 12 April 2011; Accepted 30 April 2011

Academic Editors: D. Franco and E. H. Saidi

Copyright (C 2011 Aleksander Momot. This is an open access article distributed under the Creative Commons Attribution License, which permits unrestricted use, distribution, and reproduction in any medium, provided the original work is properly cited.

Let $\Gamma \subset \mathbf{P U}(2,1)$ be a lattice which is not co-ompact, of finite covolume with respect to the Bergman metric and acting freely on the open unit ball $\mathbf{B} \subset \mathbb{C}^{2}$. Then the toroidal compactification $X=\overline{\Gamma \backslash \mathbf{B}}$ is a projective smooth surface with elliptic compactification divisor $D=X \backslash(\Gamma \backslash \mathbf{B})$. In this short note we discover a new class of unramifed ball quotients $X$. We consider ball quotients $X$ with $\operatorname{kod}(X)=1$ and $h^{1}\left(X, \mathcal{O}_{X}\right)=1$. We prove that each minimal surface with finite Mordell-Weil group in the class described admits an étale covering which is a pull-back of $X_{6}(6)$. Here $X_{6}(6)$ denotes the elliptic modular surface parametrizing elliptic curves $E$ with 6-torsion points $x, y$ which generate $E[6]$.
\end{abstract}

\section{Introduction}

Let the symbol $\tau$ denote the class of complex projective smooth surfaces $X$ which contain pairwise disjoint elliptic curves $D_{1}, \ldots, D_{h_{X}}$ such that $U=X \backslash \bigcup D_{i}$ admits the open unit ball B $\subset \mathbb{C}^{2}$ as universal holomorphic covering. As explained in [1], $\tau$ forms the "generic" class of compactified ball-quotient surfaces. There are several motivations to study surfaces in $\tau$ without assuming that the fundamental group $\pi_{1}(U, *)$ with its Poincare action on $\mathbf{B}$ is an arithmetic lattice of $\mathbf{P U}(2,1)$; we refer to [2] or to the introduction of [1]. Since the discovery of blown-up abelian surfaces in $\tau$ by Hirzebruch and Holzapfel some years ago (cf. [3]) there have been no further examples of surfaces of special type in $\tau$. In this short note we present new examples of modular surfaces $X \in \tau$ of Kodaira dimension $\operatorname{kod}(X)=1$.

In what follows we only consider complex projective smooth surfaces. An elliptic surface is an elliptic fibration $\pi: X \rightarrow C$ of a surface $X$ over a smooth curve $C$. If $\pi$ is an elliptic fibration, then a smooth fiber $F$ of $\pi$ is an elliptic curve and hence isomorphic to $\mathbb{C} /(\mathbb{Z}+\tau \mathbb{Z})$ for a $\tau$ in the upper half plane. The $j$-invariant of an elliptic surface $\pi: X \rightarrow C$ is the unique morphism from $X$ to the projective line which maps a smooth fiber $F$ of $\pi$ to $j(F)=j(\tau)$. An elliptic surface with finite Mordell-Weil group MW $(X)$ of sections is called 
extremal if the rank $\rho(X)$ of the Néron-Severi group of $X$ equals $h^{1,1}(X)$. Particular examples of elliptic surfaces arise in the following way. To each pair of positive integers

$$
(m, n) \notin\{(1,1),(1,2),(2,2),(1,3),(1,4),(2,4)\}
$$

there exists a modular surface $\pi_{n}(m): X_{n}(m) \rightarrow C_{n}(m)$ in the sense of Shioda [4] which is extremal, has no multiple fibers and nonconstant $j$-invariant. The fibration $\pi_{n}(m)$ has the following properties.

(i) The Mordell-Weil group $\mathrm{MW}\left(X_{n}(m)\right)$ is isomorphic to $\mathbb{Z} / m \mathbb{Z} \times \mathbb{Z} / n \mathbb{Z}$.

(ii) $C_{n}(m)$ is the (compactified) curve $\overline{\Gamma_{n}(m) \backslash \mathbb{H}}$ where $\Gamma_{n}(m) \subset \mathbf{S l}_{2}(\mathbb{Z})$ is the group

$$
\left\{\left(\begin{array}{ll}
a & b \\
c & d
\end{array}\right) ;\left(\begin{array}{ll}
a & b \\
c & d
\end{array}\right) \equiv\left(\begin{array}{ll}
1 & * \\
0 & 1
\end{array}\right) \bmod m, b \equiv 0 \bmod n\right\} .
$$

(iii) The curve $C_{n}(m)$ parametrizes triples $(E, x, y)$ of elliptic curves $E$ and points $x \in$ $E[m], y \in E[n]$ such that $|\mathbb{Z} x+\mathbb{Z} y|=m n$.

(iv) There are sections $\sigma_{1}, \sigma_{2}$ of order $m$, respectively, $n$ generating the Mordell-Weil group, such that a point $c \in \Gamma_{n}(m) \backslash \mathbb{H}$ corresponds to the triple

$$
E=\text { fiber over } c, \quad x=E \cap \sigma_{1}, \quad y=E \cap \sigma_{2} .
$$

(v) All singular fibers of $\pi_{n}(m)$ are of type $I_{k}$ in Kodaira's notation and they lie over the cusps of $c \in C_{n}(m)$. A representant of $c$ in $\mathbb{Q} \cup\{\infty\}$ is stabilized by a matrix $\gamma \in \Gamma$ which is an $\mathbf{S l}_{2}(\mathbb{Z})$-conjugate of

$$
\left(\begin{array}{ll}
1 & k \\
0 & 1
\end{array}\right)
$$

By [5] each extremal elliptic surface $\pi: X \rightarrow C$ with nonconstant $j$-invariant, no multiple fibers and Mordell-Weil group $\mathrm{MW}(\tilde{X})$ isomorphic to $\mathbb{Z} / m \mathbb{Z} \times \mathbb{Z} / n \mathbb{Z}$, where $(m, n)$ is as above, allows a cartesian diagram of finite maps

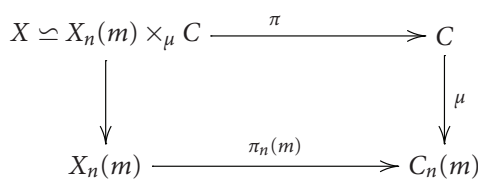

With this perspective we formulate our main result. A complex projective smooth surface $X$ is irregular if $h^{1}\left(X, \mathcal{O}_{X}\right)>0$. An irregular surface of Kodaira dimension $\operatorname{kod}(X)=1$ admits an up to isomorphism unique elliptic fibration to a curve of genus $h^{1}\left(X, \mathcal{O}_{X}\right)$. If $h^{1}\left(X, \mathcal{O}_{X}\right)=1$, then this elliptic fibration coincides with the Albanese morphism. Finally, we remark that $h^{1}\left(X_{6}(6), \mathcal{O}_{X_{6}(6)}\right)=1$ and that $C_{6}(6)$ is an elliptic curve. 
Theorem 1.1. Let $X$ be an irregular minimal surface with $\operatorname{kod}(X)=1$, elliptic fibration $\pi: X \rightarrow C$ and empty or finite Mordell-Weil group.

(1) The surface $X$ is in $\tau$ if and only if the following holds. The curve $C$ is elliptic and there exists an isogeny $v: \widetilde{C} \rightarrow C$ of elliptic curves such that $\widetilde{X}=X \times{ }_{v} \widetilde{C}$ is isomorphic to a pull-back $\mathrm{X}_{6}(6) \times{ }_{\mu} \widetilde{C}$ arising from an isogeny $\mu: \widetilde{C} \rightarrow C_{6}(6)$ with the property that $\operatorname{deg} v=6 \operatorname{deg} \mu / X(X) \leq 36$.

(2) Assume that $X$ is a surface in $\tau$ isomorphic to a pull-back $X_{6}(6) \times{ }_{\mu} \widetilde{C}$. Then the compactification divisor $D$ of $X$ consists of the 36 sections of $\pi$; each section has selfintersection number $-X(X)=-6(\operatorname{deg} \mu)$; the fibration $\pi$ admits $12(\operatorname{deg} \mu)$ singular fibers of type $I_{6}$, and each component of an $I_{6}$ intersects $D$ in precisely 6 points; one has $\rho(X)=60(\operatorname{deg} \mu)+2$ and $X$ is extremal.

\section{Some Basic Properties of Surfaces in $\tau$}

We cite two results on ball-quotient surfaces which will be needed for the proof of the theorem. The first result is essentially [6, Theorem 3.1] restricted to $\operatorname{dim} X=2$ with attention to sign conventions, except the assertion on semistability. The latter assertion follows from [7]. A reduced effective divisor on a surface $X$ is called semistable if it has normal crossings and if every rational smooth prime component intersects the remaining components in more than one point. If $T, D$ are divisors on $X$, we say that $T$ is ample modulo $D$ if $T^{2}>0$ and if the intersection number $C T$ is positive for each curve $C$ on $X$ not supported in $D$.

Theorem 2.1 (see $[6,7]$ ). Let $X$ be a smooth projective surface and $D \subset X$ a divisor with normal crossings. Suppose that $K_{X}+D$ is big and ample modulo D. Then

$$
c_{1}^{2}\left(\Omega_{X}^{1}(\log D)\right) \leq 3 c_{2}\left(\Omega_{X}^{1}(\log D)\right)
$$

with equality if and only if $X \backslash D$ is an unramified ball quotient $\Gamma \backslash \mathbf{B}$ and $D$ is semistable.

There is a canonical exact sequence

$$
0 \longrightarrow \Omega_{X}^{1} \longrightarrow \Omega_{X}^{1}(\log D) \stackrel{\text { res }}{\longrightarrow} \mathcal{O}_{D} \longrightarrow 0
$$

where res is the Poincaré residue map. With this one proves that $c_{1}\left(\Omega_{X}^{1}(\log D)\right)=[D]-$ $c_{1}(X) \in H^{2}(X, \mathbb{C})$ and $c_{2}\left(\Omega_{X}^{1}(\log D)\right)=c_{2}(X)-\left(c_{1}(X),[D]\right)+([D],[D]) \in H^{4}(X, \mathbb{C})$. Therefore,

$$
c_{1}^{2}\left(\Omega_{X}^{1}(\log D)\right)=\left(K_{X}+D\right)^{2} .
$$

If $\Gamma^{\prime} \subset \Gamma$ is a neat normal subgroup with finite index in $\Gamma$, then $\Gamma^{\prime} \backslash \mathbf{B}$ is compactified by a smooth elliptic divisor, and $\Gamma \backslash \mathbf{B}$ is compactified by a divisor $D$. As $D$ is the quotient $D^{\prime} / G$, where $G=\Gamma / \Gamma^{\prime}$, it is a normal curve. Hence, $D$ is smooth and consists of elliptic curves, for rational curves cannot appear because of semistability. Thus, if equality holds in the theorem, then $D$ is smooth. Moreover, one can show that $K_{X}+D$ is big and ample modulo $D$. 
Lemma 2.2. Let $X$ be in $\tau$ with compactification divisor $D$ and consider an irreducible curve $L \subset X$. If $L$ is smooth rational, then $|L \cap D| \geq 3$. And if $L$ is a smooth elliptic curve, then $|L \cap D| \geq 1$.

Proof. If the statement is wrong for some rational curve $L$, then the induced holomorphic map of universal coverings $\widetilde{L \backslash D} \rightarrow \mathbf{B}=\widetilde{X \backslash D}$ yields a contradiction to Liouville's theorem.

\section{Proof of the Theorem}

We begin by proving (1) and suppose that $X$ is an irregular minimal surface of Kodaira dimension $\operatorname{kod}(X)=1$ in $\tau$. Then $X$ is an elliptic surface in a unique way. We denote by $\pi: X \rightarrow C$ the elliptic fibration and assume that its Mordell-Weil group is empty or finite. As above, $D$ is the compactification divisor of $X$. Moreover, we let $F$ be the numerical class of a fiber of $\pi$. Since $K_{X}+D$ is ample modulo $D$, it follows that $F$ has positive intersection with $D$. Thus, a component of $D$ dominates $C$. The theorem of Hurwitz implies that $C$ is an elliptic curve and $h^{1}\left(X, \mathcal{O}_{X}\right)=1$. Moreover, after transition to an etale cover $v: \widetilde{C} \rightarrow C$ and performing a base change $\tilde{X}=X \times{ }_{v} \widetilde{C}$, we achieve that every $\widetilde{D}_{i}$ is a section as soon as it dominates $\tilde{C}[1$, Lemma 3.3]. We will assume for the time being that $X=\tilde{X}$; once we have shown that (1) is true if $X=\tilde{X}$, it will be easy to obtain (1) in the general case. If $X=\tilde{X}$, then the curves $D_{i}$ are all sections, because they are disjoint. In this case we have the following.

Lemma 3.1. The identities $36 x(X)=D F \cdot X(X)=-D^{2}$ and $D F=36$ hold.

Proof. The canonical bundle formula implies that $K_{X}=\pi^{*}(\mathfrak{c})$ with a Weil divisor $\mathfrak{c} \in \operatorname{Div}(C)$. Moreover, $h^{0}\left(X, m K_{X}\right)=h^{0}(C, m c)$. The theorem of Riemann-Roch yields $h^{0}\left(X, K_{X}\right)=$ $\operatorname{deg} \mathfrak{c}>0$. It results from the adjunction formula that

$$
D_{i}^{2}=-\operatorname{deg} \mathfrak{c}=-h^{0}\left(X, K_{X}\right)=-\chi(X)
$$

Hence, $-D^{2}=-\sum D_{i}^{2}=D F X(X)$. Furthermore, $12 X(X)=c_{2}(X)$ by Noether's formula. So, Theorem 2.1 yields the remaining identities.

We consider the Mordell-Weil group $\operatorname{MW}(X)=\operatorname{MW}(\tilde{X})$. By assumption, $\operatorname{MW}(X)$ is finite and equals $\mathrm{MW}_{\text {tor }}(X)$. It follows from the previous lemma that $\left|\mathrm{MW}_{\text {tor }}(X)\right| \geq D F=36$. We next prove the following lemma of general interest.

Lemma 3.2. Let $\pi: X \rightarrow C$ be a minimal elliptic surface over an elliptic curve $C$. Assume that $\operatorname{kod}(X) \geq 1$ and that each rational curve $L \subset X$ intersects at least one section of $\pi$. Suppose moreover that $\left|\mathrm{MW}_{\text {tor }}(X)\right| \geq 33$. Then the following assertions hold.

(1) $\mathrm{MW}(X)$ is a torsion group isomorphic to $\mathbb{Z} / 6 \mathbb{Z} \times \mathbb{Z} / 6 \mathbb{Z}$.

(2) All singular fibers of $\pi$ are semistable of type $I_{6}$ and each rational curve $L \subset I_{n}$ intersects 6 distinct sections of $\pi$.

(3) $X$ has $2 X(X)$ singular fibers.

(4) $\rho(X)=h^{1,1}(X)=10 \chi(X)+2$. 
Proof. Statement (1) follows directly from [8, equation (4.8)]. If $\mathrm{MW}(X)$ is a torsion group, then its sections do not intersect each other. So, their sum is a smooth divisor $D$. Moreover, [8, Lemma 1.1] implies that all singular fibers are of type $I_{n}$ for some $n>0$. If $H_{n} \subset M(X)$ is the nontrivial isotropy group of a node $x \in I_{n}$ then $H_{n}$ and $\mathrm{MW}_{\text {tor }}(X) / H_{n}$ are cyclic by [8, Lemma 2.2]. Because of (1) we thus have $\left|H_{n}\right|=6$ for all isotropy groups $H_{n}$. Let $S \in$ $\operatorname{MW}(X)$ be the neutral section. By the proof of [8, Lemma 2.2], $H_{n}$ consists of those sections which intersect the prime component $L \subset I_{n}$ containing $S \cap I_{n}$. However, since by assumption we may take any section to be the neutral element of $M W(X)$, we have $L D=6$ for each component $L \subset I_{n}$. As $D I_{n}=36$, we get $n=6$. This yields (2). Recalling that $\sum_{I_{n}} n=c_{2}(X)$, we find for the number $t$ of singular fibers of $\pi$

$$
t=2 \chi(X)=2 g(C)-2+\operatorname{rank} \operatorname{MW}(X)+2 \chi(X) .
$$

We receive (3). Finally, according to [8, Proposition 1.6] the last equality holds if and only if $\rho(X)=h^{1,1}(X)$, so that $X$ is extremal. An easy calculation shows now (4).

As explained in Section 1, $X$ is isomorphic to a pull-back $X_{6}(6) \times{ }_{\mu} C$. This shows (1) in the theorem if $X=\tilde{X}$. Next we withdraw the additional assumption that $X=\tilde{X}$ from the beginning of the proof and let $v: \widetilde{C} \rightarrow C$ be an isogeny of minimal degree such that $\tilde{X}$ is a pull-back $X_{6}(6) \times{ }_{\mu} C$. We are left to show that $\operatorname{deg} v \leq 36$ and $X(X) \cdot \operatorname{deg} v=6 \operatorname{deg} \mu$. The former estimate is clear, because over a curve $D_{i} \subset X$ there lie $\leq 36$ curves $\tilde{D}_{i} \subset \tilde{X}$. The latter equality holds, because $X(X)=6 \operatorname{deg} \mu / \operatorname{deg} v$ by the lemma below. This yields (1) in the general case. Statement (2) in the theorem results from Lemma 3.2, the fact that $\mu$ is étale and the following lemma.

Lemma 3.3. The modular surface $X_{6}(6)$ has invariant $\chi\left(X_{6}(6)\right)=6$.

Proof. This is a consequence of the formulae in $[9$, page $77 \mathrm{f}]$.

\section{References}

[1] A. Momot, "Irregular ball-quotient surfaces with non-positive Kodaira dimension," Mathematical Research Letters, vol. 15, no. 6, pp. 1187-1195, 2008.

[2] R.-P. Holzapfel, Ball and Surface Arithmetics, vol. E29 of Aspects of Mathematics, Friedr. Vieweg \& Sohn, Braunschweig, Germany, 1998.

[3] R.-P. Holzapfel, "Jacobi theta embedding of a hyperbolic 4-space with cusps," in Geometry, Integrability and Quantization, pp. 11-63, Coral Press Science, Sofia, Bulgaria, 2002.

[4] T. Shioda, "On elliptic modular surfaces," Journal of the Mathematical Society of Japan, vol. 24, pp. 20-59, 1972.

[5] R. Kloosterman, "Extremal elliptic surfaces and infinitesimal Torelli," The Michigan Mathematical Journal, vol. 52, no. 1, pp. 141-161, 2004.

[6] G. Tian and S.-T. Yau, "Existence of Kähler-Einstein metrics on complete Kähler manifolds and their applications to algebraic geometry," in Mathematical Aspects of String Theory, vol. 1 of Adv. Ser. Math. Phys., pp. 574-628, World Science Publishing, Singapore, 1987.

[7] Y. Miyaoka, "The maximal number of quotient singularities on surfaces with given numerical invariants," Mathematische Annalen, vol. 268, no. 2, pp. 159-171, 1984.

[8] R. Miranda and U. Persson, "Torsion groups of elliptic surfaces," Compositio Mathematica, vol. 72, no. 3, pp. 249-267, 1989.

[9] W. Barth and K. Hulek, "Projective models of Shioda modular surfaces," Manuscripta Mathematica, vol. 50, pp. 73-132, 1985. 


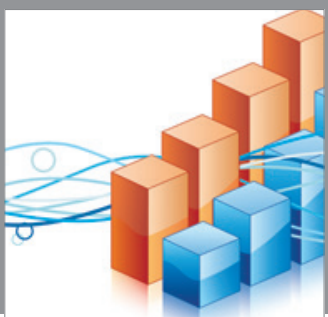

Advances in

Operations Research

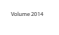

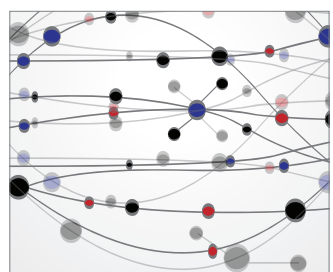

\section{The Scientific} World Journal
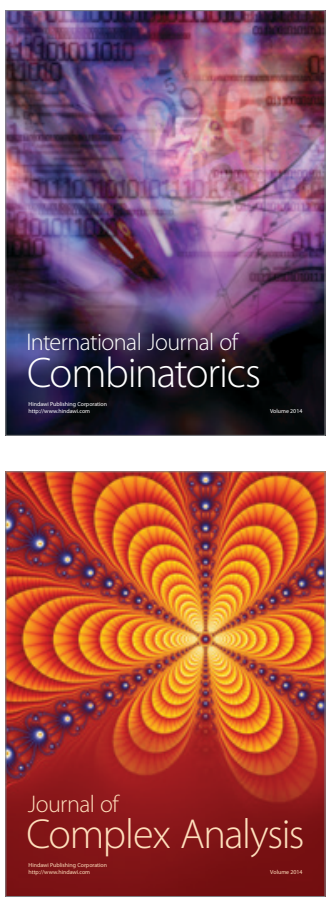

International Journal of

Mathematics and

Mathematical

Sciences
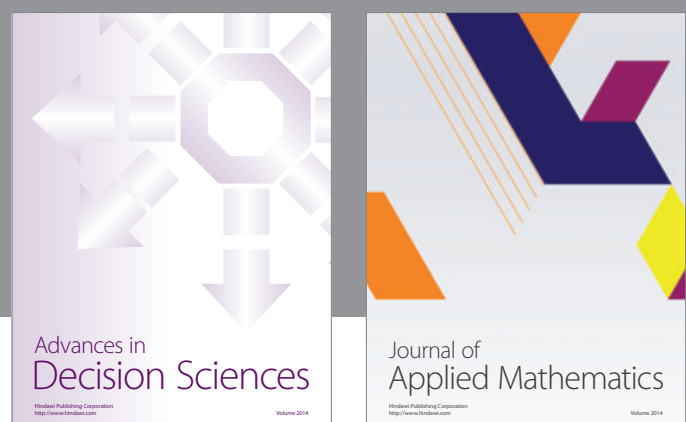

Journal of

Applied Mathematics
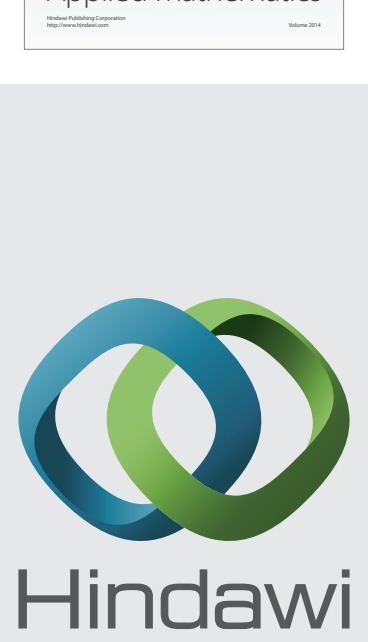

Submit your manuscripts at http://www.hindawi.com
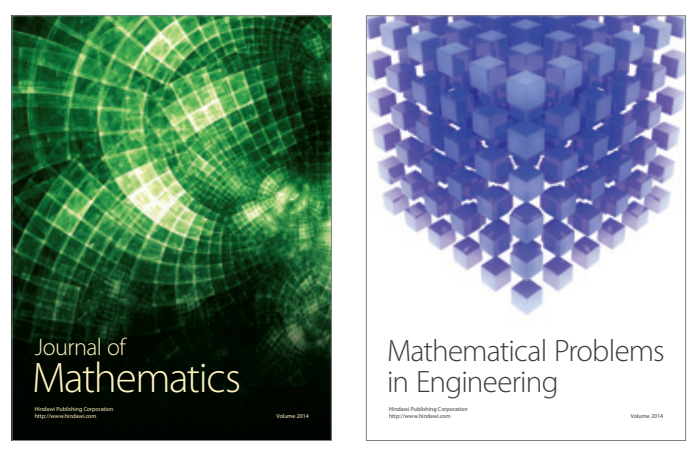

Mathematical Problems in Engineering
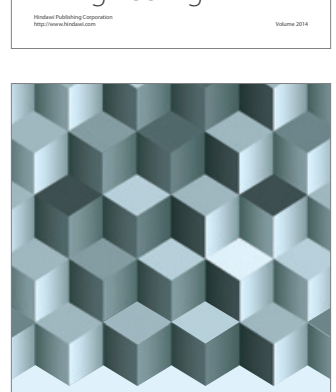

Journal of

Function Spaces
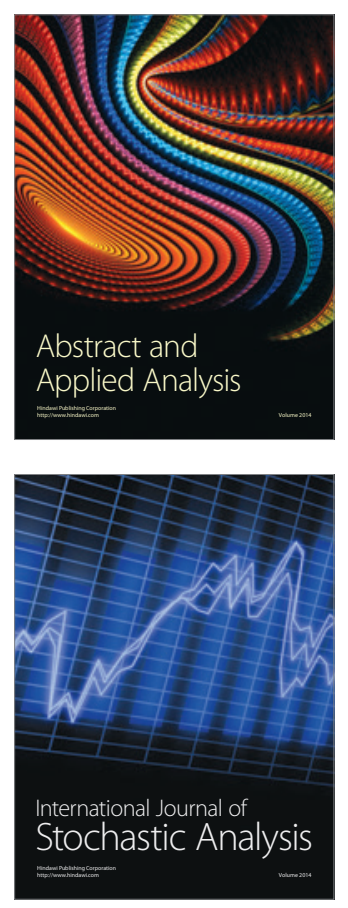

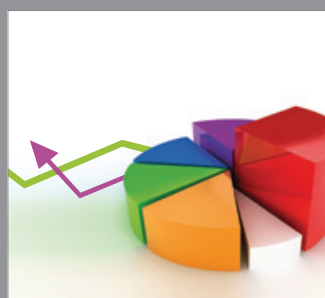

ournal of

Probability and Statistics

Promensencen
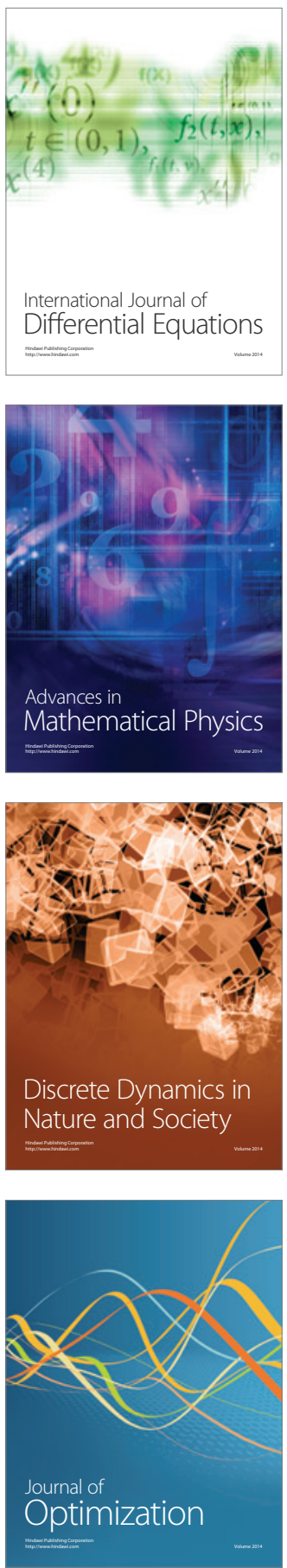